\title{
A Test Method for Helicopter Landing Gear Load
}

\author{
Jiahong Zheng \\ Institute of Flight Test Technology, Chinese Flight Test Establishment, Xi'an, China
}

Email address:

040715316@163.com

\section{To cite this article:}

Jiahong Zheng. A Test Method for Helicopter Landing Gear Load. International Journal of Mechanical Engineering and Applications. Vol. 9, No. 1, 2021, pp. 19-24. doi: 10.11648/j.ijmea.20210901.14

Received: January 18, 2021; Accepted: March 3, 2021; Published: March 4, 2021

\begin{abstract}
In this paper, a load test method of landing gear during take-off and landing of helicopter was proposed and the structure of helicopter landing gear, the modification of strain gauge bridge, the establishment of load calibration equation and the flight test of helicopter were studied. Firstly, according to the structure of helicopter landing gear, the stress analysis of landing gear was carried out, and the load position and force form of landing gear were determined during take-off and landing. Then, the measuring principle of the strain bridge was studied. According to the technical requirements, structural characteristics of the landing gear load test and the properties and principles of the strain bridge, the distribution and load measurement form of the strain bridge of the dangerous structure of the landing gear were designed. Then, studying load calibration test scheme of landing gear and carrying out load calibration test, the load calibration equation of landing gear was obtained, and the accuracy of load calibration equation was verified by comparing the expected load and actual load. Through the flight test, the landing gear load during the take-off and landing of the helicopter was obtained. Finally, the valid analysis of flight load data was carried out. The results show that the proposed method is feasible, and the flight test data of landing gear load are accurate and reliable, which can provide data support for the structure modification, fatigue analysis and the life evaluation of landing gear.
\end{abstract}

Keywords: Helicopter, Landing Gear, Flight Test, Load Measurement

\section{Introduction}

Helicopter landing gear is one of the most important bearing parts of helicopter. The main function of landing gear is to absorb the energy during landing on or taking off, and reduces the overload caused by landing impact. In addition, landing gear is often used to enable helicopters to glide and run on the ground, and to reduce the impact and turbulence caused by uneven ground. Therefore, the stress condition and structural deformation of landing gear of helicopter during take-off, landing and sliding has a tight relationship with the safety of helicopter structure. It is necessary to measure the loads of the helicopter landing gear in the condition of taking off, landing and sliding, so as to provide the support for the design and life evaluation of the helicopter landing gear [1-3].

The purpose of the flight test of helicopter landing gear load is to verify the structural design load of helicopter landing gear and to provide the basis for analyzing and evaluating the structural strength of helicopter landing gear. The load measurement of helicopter landing gear is generally the implementation project when a new helicopter is designed or modified. There are requirements for helicopter landing gear loads in relevant helicopter specifications, such as the description of in 5.3.1 of GJB5771-2006"General requirements for shipborne helicopters" that helicopter landing gear systems should fully absorb the impact energy at a sinking speed of not less than $3.05 \mathrm{~m} / \mathrm{s}$ without permanent deformation and the structure will not be destroyed with 1.5 times the above impact energy [4]. There are requirements for the maximum use of sinking speed for the use of a certain helicopter. Therefore, it is necessary to measure the landing gear landing load and give the test results.

At present, many researchers have used mathematical models to study landing gear flight loads [3, 5-7]. However, there are lack of researches on landing gear flight load and damage accumulation at home and abroad and flight test data. On the other hand, the load of landing gear cannot be fully simulated in the laboratory, so it is difficult to accurately evaluate the life of landing gear.

In this paper, we discuss the structure form of helicopter landing gear, the modification of strain gages and the 
establishment of load calibration equation, and the method was applied to the load flight test of helicopter landing gear. Through the analysis of the flight test data, the results show that the method is feasible, and the flight test data of landing gear load are accurate and reliable, and the data can be used in landing gear life evaluation.

\section{Load Analysis of Landing Gear}

In this paper, the configuration of the left landing gear was analyzed. The structure of this landing gear is mainly composed of buffer prop, anti-torsion arm, wheel shaft, inner cylinder, outer cylinder, tire, brake device and so on. The structure of the landing gear is shown in figure 1.

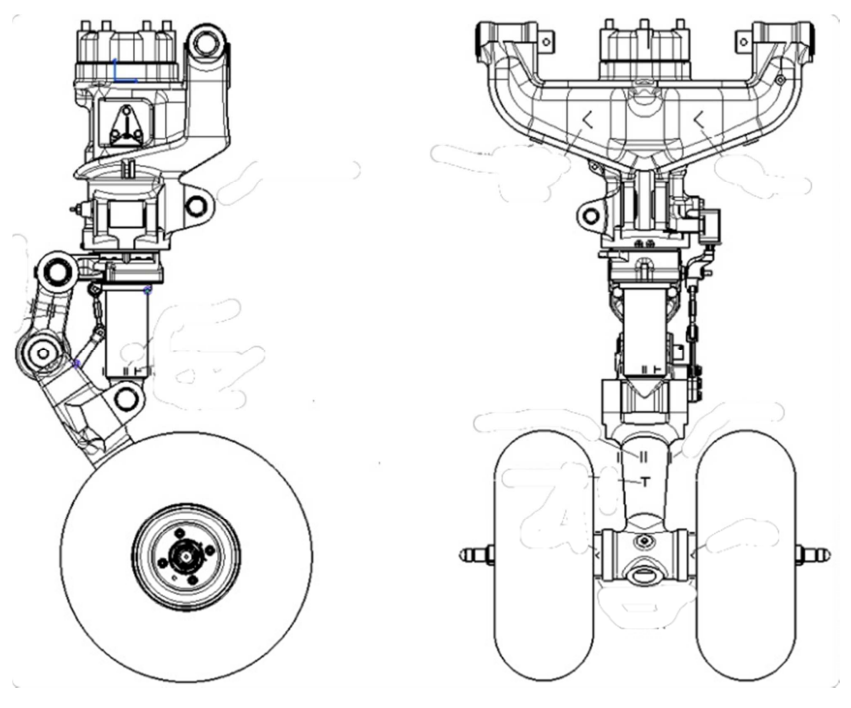

Figure 1. The structure of landing gear.

In the condition of take-off, landing and ground handling, the landing gear usually bears on three directions of load, namely, heading force $p_{x}$, lateral force $p_{y}$ and vertical force $p_{z}$, which lead to the deformation of the landing gear structure. In the action of vertical force, the wheel shaft is subjected to bending moment and shear, and the rocker arm is subjected to bending moment and axial force, and the anti-torsion arm is subjected to the tension and bending moment, and the inner cylinder is subjected to the axial force. In the action of heading force, the wheel shaft is subjected to bending moment and shear, and the rocker arm is subjected to bending moment, and the anti-torsion arm and inner cylinder are subjected to the bending moment and axial force. In the action of lateral force, the rocker arm and the outer cylinder are subjected to bending moment.

According to the superposition principle, when the landing gear structure works in the elastic range, the output of the strain gauge bridge is linearly related to the external load, and the strain equation can be denoted as follow.

$$
\mathcal{E}=a_{1} p_{x}+a_{2} p_{y}+a_{3} p_{z}
$$

Where $\varepsilon$ is the strain caused by landing gear loads, and $a_{1}, a_{2}, a_{3}$ are the coefficient.
When the landing gear load is measured, we require an external load, so it is assumed that in a buffer prop travel state, for $i$ bridge, $j$ kinds of calibration loads are applied. The load equation can be expressed as follows.

$$
\left[\begin{array}{cccc}
p_{x 1} & p_{x 2} & \cdots & p_{x j} \\
p_{y 1} & p_{y 2} & \cdots & p_{y j} \\
p_{z 1} & p_{z 2} & \cdots & p_{z j}
\end{array}\right]=\left[\begin{array}{llll}
b_{11} & b_{12} & \cdots & b_{1 i} \\
b_{21} & b_{22} & \cdots & b_{2 i} \\
b_{31} & b_{32} & \cdots & b_{3 i}
\end{array}\right]\left[\begin{array}{cccc}
\varepsilon_{11} & \varepsilon_{12} & \cdots & \varepsilon_{1 j} \\
\varepsilon_{21} & \varepsilon_{22} & \cdots & \varepsilon_{2 j} \\
\vdots & \vdots & \cdots & \vdots \\
\varepsilon_{i 1} & \varepsilon_{i 2} & \cdots & \varepsilon_{i j}
\end{array}\right]
$$

It can be seen from the load equation that the load on the landing gear and the output value of the strain bridge are multivariate linear regression relations [8-10].

\section{The Principle of Flight Load Test and Modification}

\subsection{Strain Electrotometry}

(a) the principle of strain gauges

Using the strain-resistance effect of wire, the strain on the surface of the componentis directly transformed into the relative change of resistance, so that the strain measurement can be carried out by electrical measurement. The strain gauge is a sensor made by this principle. The relationship between strain and relative variation of resistance can be expressed as follow.

$$
\frac{d R}{R}=K \varepsilon
$$

where $\frac{d R}{R}$ is the relative variation of resistance, $K$ the sensitivity coefficient of strain gauge wire and $\varepsilon$ is the strain of the component.

(b) Bridge measuring circuit

During the load test of landing gear, the strain on the surface of the component is converted into $\Delta \mathrm{R} / \mathrm{R}$ by sticking a strain gauge on the surface of the component. Because the change of strain and strain resistance is generally very small, it is difficult to measure directly and accurately. Therefore, it is necessary to use a full bridge measuring circuit to convert the small change of the resistance value of the strain gauge into a voltage signal. At the same time, the value of the indicated strain can be increased. Full bridge measuring circuit is connected with resistance strain gauge on four bridge arms, and the sensitivity coefficient is $K$. The output voltage is obtained by the follow formula [11].

$$
U_{0}=\frac{u}{4} k\left(\varepsilon_{1}-\varepsilon_{2}+\varepsilon_{3}-\varepsilon_{4}\right)=u k \varepsilon
$$

Then the strain of the output is as follow.

$$
\varepsilon=\frac{u_{0}}{k u}
$$

\subsection{Configuration ofStrain Bridge and Location of Measuring}

The strain gages are pasted in the region with large strain 
and no stress concentration, and the strain gages should avoid obstacles, strain force concentration and section with too large stress gradient to ensure the linearity and reliability of the data. Using the whole bridge measurement method not only can eliminate the influence of the temperature effect caused by the different paste position on the output accuracy of the bridge, but also can improve the voltage amplitude of the output signal and increase the sensitivity of the output signal and reduce the interference of irrelevant signals. According to the technical requirements of landing gear load test and its structural characteristics and combined with the properties and principles of the bridge measuring circuit, the determination of the measured load amount was carried out on each key component of landing gear, such as the bending moment of the anti-torsion arm, the tension force of the buffer and so on.

a) Two measuring points on anti-torsion arm of the landing gear were shown in Figure 2.

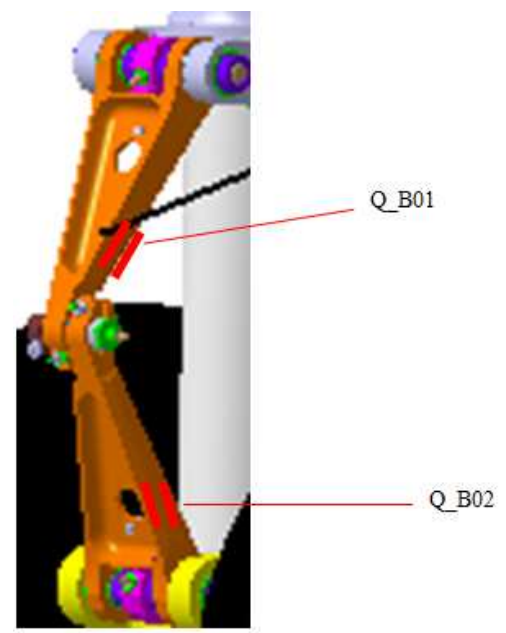

Figure 2. Measuring points of the front landing gear arm.

Where bending strain of front arm is Q_B01 and bending strain of front arm is Q_B02.

b) Schematic illustration of front landing gear, bottom of inner tube and outer tube patch was shown in Figure 3.
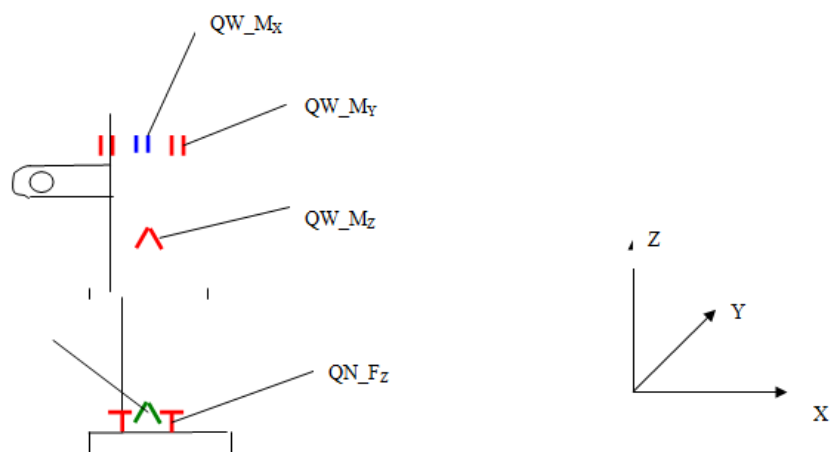

Figure 3. Measuring points of the outer tube and inner tube.

Where QW MX is the bending strain in X direction of the outer cylinder. QW_MY is the bending strain in $\mathrm{Y}$ direction of the outer cylinder. QW_MZ is the bending strain in $\mathrm{Z}$ direction of the outer cylinder. QN_MZ is the bending strain in $Z$ direction from the inner cylinder. QN_FZ is the Stretch strain in $\mathrm{Z}$ direction of the inner cylinder.

c) Schematic illustration of the patch on the landing gear wheel axle (left and right symmetrical from the reverse direction,) was shown in Figure 4.

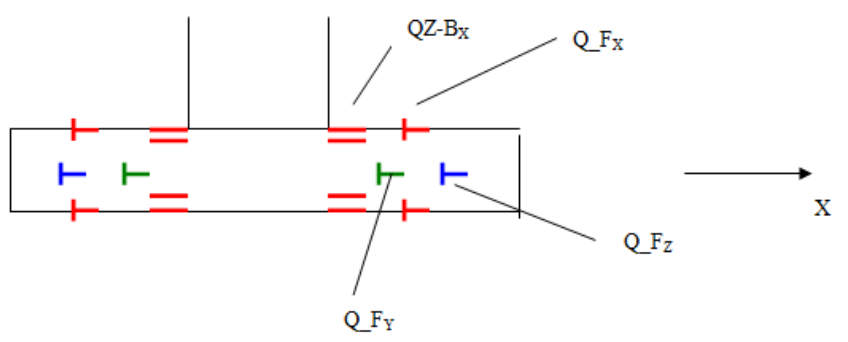

Figure 4. Measuring points of the landing gear wheel axle.

Where QZ_BX1 is X direction bending strain for the front landing gear wheel axle. QZ_BX2 is X direction bending strain for the front landing gear wheel axle. QZ_FX1 is the Stretch strain in $\mathrm{X}$ direction for the front landing gear wheel axle. QZ_FX2 is Stretch strain 2 in X direction for the front landing gear wheel axle. QZ_FZ1 is the Stretch strain for Z direction for the front landing gear wheel axle. QZ_FZ2 is the stretch strain in $\mathrm{Z}$ direction for the front landing gear wheel axle.

QZ_FY1 axle is the Stretch strain in Y direction for the front landing gear wheel axle. QZ_FY2 is the stretch strain in $\mathrm{Y}$ direction for the front landing gear wheel axle.

\subsection{Load Calibration Test for Landing Gear}

In the process of development and use of helicopter, it is necessary to carry out load calibration test on structural componentsand establish a quantitative relationship between input load and strain bridge, that is, load calibration equation. Applying the load calibration equation to the measured strain, the strain time course can be converted into load time course. This is the premise of strength design, reliability analysis and fatigue test. When the landing gear is subjected to loads, its stress is in the elastic range. Therefore, the landing gear load calibration equation can be obtained by multivariate linear regression method as mentioned above.

The landing gear load calibration can be carried out by platform loading calibration and bench calibration. The platform loading cannot remove the landing gear and fix the helicopter with the landing gear on the loading platform for vertical, heading and lateral loading. The disadvantages of this way are that the vertical load range is small, the heading and lateral unidirectional loading cannot be carried out, and the loading fixed balance is complex, and the loading implementation is difficult. However, the bench calibration overcomes the above shortcomings, can be one-way, two-way and three-way loading, and loading implementation is more convenient. Therefore, this paper adopts bench loading calibration.

The load ground static calibration test was carried out to obtain the relation between load and strain. The strain gauges were calibrated jointly with the test equipment to reduce the system error. 


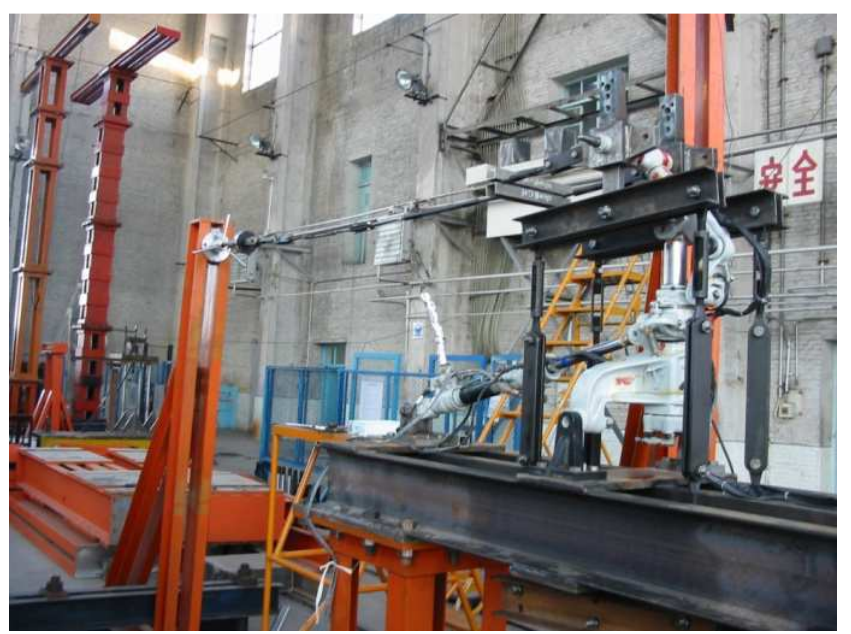

Figure 5. Load calibration of the front landing gear.

In the test, the landing gear simulated the real connection mode. The landing gear was fixed on the special fixture, and the gas in the buffer was replaced by oil. By adjusting the amount of oil to meet the compression of the buffer in each state. Three directions were loaded. The Load calibration of the landing gear can be seen in figure 5. According to the regression processing of load calibration test data, the calibration equations of the heading load, the vertical load and the lateral load of front landing gear were obtained.

In order to verify the accuracy of the load calibration equation, the static strain gauge was used to measure the
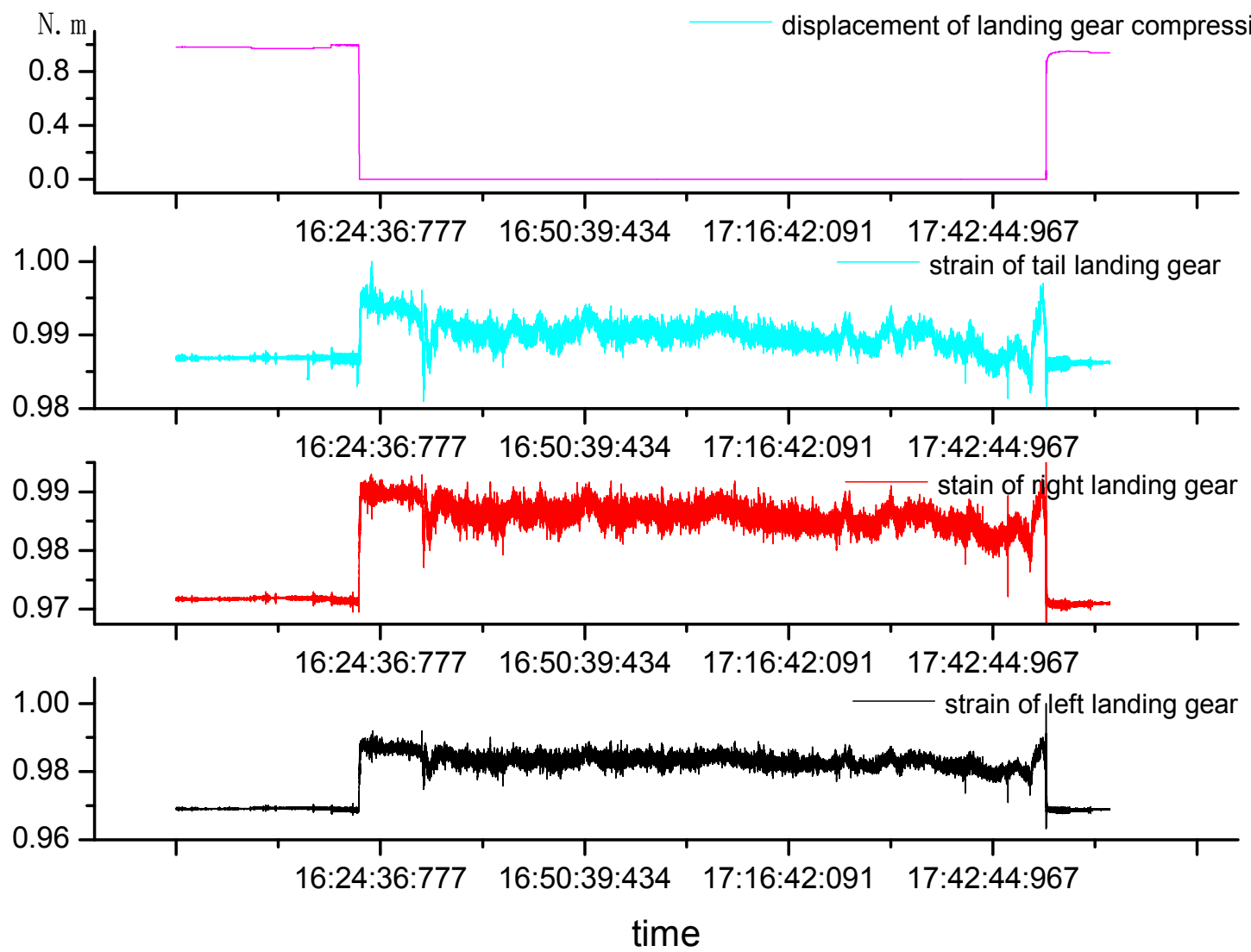

Figure 6. Load time course of landing gear. gear, and the load equation was substituted to obtain the calculated value. The accuracy of the equation was verified by the expected load and the actual load. After verification, the relative error between the load equation and the actual load is less than 5\%, which meets the requirements [12-16].

\subsection{Testing Equipment} were transmitted to the airborne information acquisition system KAM500 by connecting the cable. The system adjusted the output analog signals of the strain gages mounted on each arm into digital quantity and collected them into the memory card.

\subsection{Test Flight Method} with different center of gravity. The parameters such as time, altitude of helicopter, attitude angle of landing, sinking speed of landing, gliding speed, turning radius, turning speed and moving speed were measured in flight test.

\section{Test Results and Analysis}

\subsection{Test Data Processing Analysis Methods}

Flight test data processing methods include data preprocessing phase and data secondary processing phase.

strain of each channel under the actual load of the landing

The signals of strain gages mounted on the landing gear

During the flight test, the helicopter takes off and glides$$
\text { 年 }
$$ 
a) Data preprocessing

Firstly, determine the validity of flight data. secondly, select the time period corresponding to the flight state and the load data corresponding to that time period. Finally, remove abnormal data through softwares and programs.

b) Data secondary processing stage

According to the task requirements, the load test parameters in the corresponding time period of the flight test subject were calculated and analyzed. The dynamic and static loads are included in the measured load signals, and the separation of dynamic and static values from the measured signals is the essential to the fatigue spectrum compilation and life evaluation of landing gear. According to the theoretical of landing gear load generation and the characteristics of the measured load signal, the static load measurement value was given by time domain average analysis, and the load cycle statistics was carried out by non-cyclic counting method. The dynamic load (half amplitude) measurement value was obtained. The calculation formulas are as follow $[11,14,17]$.

$$
\begin{aligned}
& \text { Dynamic }=\frac{\text { Max }- \text { Min }}{2} \\
& \text { Static }=\frac{\text { Max }+ \text { Min }}{2}
\end{aligned}
$$

\subsection{Test Results and Analysis}

As shown in figure 6, it was the time domain diagram of landing gear load for helicopter's normal center of gravity position during the process of landing and then taking off. It can be clearly seen from the diagram that the waveform was continuous without clutter interference and jumping point, and it indicates that the load transmission signal is normal. From the time domain waveform of the whole helicopter landing, it can be seen that when the helicopter takes off, the landing gear load decreases. when the helicopter lands, the landing gear load increases, which accords with the theoretical analysis.

\section{Conclusion}

This paper presents a load test method for landing gear during take-off and landing of helicopter. In this paper, the configuration of the left landing gear was analyzed, and it can be drawn that the load on the landing gear and the output of the strain bridge are multiple linear regression relations when the landing gear works in the elastic range. The measuring principle of strain bridge was studied, and the whole bridge measurement method was adopted for the measurement of landing gear, which not only can eliminate the influence of temperature caused by different adhesive position, but also can improve the voltage amplitude of output signal, and increase the sensitivity of output signal and reduce the interference of signals. The load calibration test of landing gear was studied, and the original data of load calibration test were obtained. Then, the calibration equations of heading load, vertical load and lateral load of landing gear were obtained by the regression of load calibration test data. The helicopter took off and slid at different centers of gravity. The flight parameters such as height, speed, attitudes were measured during flight test. Finally, the data processing method was introduced, and the validity of take-off and landing load data was analyzed. The results show that the helicopter landing gear load measurement scheme is feasible, and the data is accurate and reliable, which can meet the precision requirements. This method can be applied to load measurement of other helicopter components.

\section{Recommendations for Future Work}

This work based on the principle of strain bridge, and the take-off and landing loads of landing gear were obtained by sticking strain gauge in dangerous positions of landing gear. In recent years, fiber has been widely used in strain measurement because of its advantages of strong signal interference resistance, convenient installation and high measurement accuracy [18]. Therefore, it is suggested that fiber is used to measure the load of landing gear in the future, and the results can be compared and analyzed.

\section{Acknowledgements}

This work was supported by the Department of Naval Equipment Aviation of China for the development of a type of helicopter.

\section{References}

[1] $\mathrm{Mu}$ Zhitao, and ZengBenyin, Helicopter Structure Fatigue. Beijing, CHN: National Defence Industry Press, 2009, pp. 1-3.

[2] Sun Zhizhao, XuGuiqi, Xiao Qiuting, The strength of helicopter, Beijing: Aviation Industry Press, 1990. 11: 151-152.

[3] ZbigniewSkorupka. Laboratory Investigations on Landing Gear Ground Reactions (Load) Measurement. Journal of KONES. 2017, 24 (2): 225-230.

[4] Kou Fujun. The load measurement technology of landing gear in helicopter. The Twenty-eight. Annual Session of Helicopter. Shangyu, China, 2012, pp: 100-106.

[5] TianJiajie, Mu Po, Cheng Suhua, Feng Xi. Drop Simulation and Drop Test of Aircraft Main Landing Gear with High Sinking Velocity. Chinese Hydraulics and Pneumatics, 2020, (10): 174-180.

[6] Huaitao Wang, Dafang Wu, Feng Wang, HaoyuanRen. A method for determining the horizontal impact load based on the rotational speed of the aircraft's wheel in a landing gear drop test. International Journal of Crashworthiness, 2018, 23 (6): 627-634.

[7] ZuoYihong, HE Hongli, FENG Qiaoning, Tian Wei-feng. Application of High Speed Video in Load Test for Aircraft Landing Gear. Electro-opticatechnology Application. 2011, 26 (3): $82-84$. 
[8] Currey N S. Aircraft landing gear design: principles and practice. Washington, D. C.: AIAA, 1998: 1-20.

[9] Cao jingtao. The load measurement technology of landing gear in aircraft. Applied Technology, 2014; 6 (15): 176-178.

[10] Fang Kaitai, QuanHui, Chen Qingyun, the analysis of Regression [M], Science Press, Beijing, 1988: 64-69.

[11] Kligko M P, Aphaytob E B. Aircraft strength flight test (static). xi'an: ASST System. Engineering Office of Ministry of Aeronautics and Astronautics, 1992. 23 26.

[12] Kadarno P, Barrinaya M. A, Manurung A. O. Mechanism analysis of a main landing gear of transporting aircraft: A design learning perspective. Engineering Failure Analysis. $2021,119$.

[13] AdityaArmaan, Sharma Keshav, G. Srinivas. A step towards safety: Material failure analysis of landing gear. Materials Today: Proceedings. 2020, 27 (1): 402-409.
[14] Li Yongshou, Tang Lifang. Study on Load Measurement of Transmission System in Helicopter Flight. Science and Technology Innovation Herald. 2011, 6: 2-3.

[15] Wu Wei, Zhang Shuming, Teng Qi, Yan Chuliang. Study on load measurement and calibration test for airplane landing gear. Journal of Mechanical Strength, 2003 (01): 48-51.

[16] Tang Ani. Analysis of usage landing gears loads spectrum for an aircraft's landing gears. Structure and Environment Engineering. 2007, 34 (4): 43-48.

[17] GAO Zhengtong. Fatigue application statistics. Beijing: National Defense Industry Press, 1986. 336 344.

[18] A. Iele, M. Leone, M. Consales, G. V. Persiano. Load monitoring of aircraft landing gears using fiber optic sensors. Sensors and Actuators A: Physical, 2018, 281: 31-41. 